Bartsch, Silke; Henke, Katharina; Müller, Heike; Penning, Isabelle

\title{
Verbraucherkompetenzen für morgen durch Lehrkräftebildung heute: Professionalisierung von Lehrpersonen in der Verbraucherbildung
}

Haushalt in Bildung \& Forschung 8 (2019) 4, S. 90-103

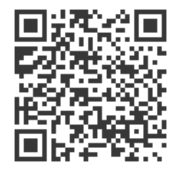

Quellenangabe/ Reference:

Bartsch, Silke; Henke, Katharina; Müller, Heike; Penning, Isabelle: Verbraucherkompetenzen für morgen durch Lehrkräftebildung heute: Professionalisierung von Lehrpersonen in der Verbraucherbildung - In: Haushalt in Bildung \& Forschung 8 (2019) 4, S. 90-103 - URN: urn:nbn:de:0111-pedocs-236509 - DOI: 10.25656/01:23650

https://nbn-resolving.org/urn:nbn:de:0111-pedocs-236509

https://doi.org/10.25656/01:23650

in Kooperation mit / in cooperation with:

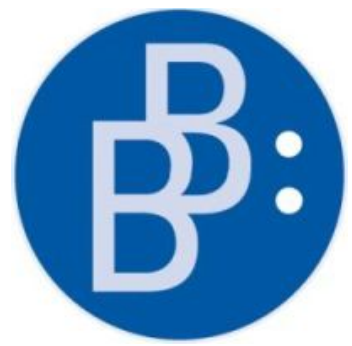

https://www.budrich.de

\section{Nutzungsbedingungen}

Dieses Dokument steht unter folgender Creative Commons-Lizenz: http://creativecommons.org/licenses/by-sa/4.0/deed.de - Sie dürfen das Werk bzw. den Inhalt vervielfältigen, verbreiten und öffentlich zugänglich machen sowie Abwandlungen und Bearbeitungen des Werkes bzw. Inhaltes anfertigen, solange sie den Namen des Autors/Rechteinhabers in der von ihm festgelegten Weise nennen und die daraufhin neu entstandenen Werke bzw. Inhalte nur unter Verwendung von Lizenzbedingungen weitergeben, die mit denen dieses Lizenzvertrags identisch, vergleichbar oder kompatibel sind.

Mit der Verwendung dieses Dokuments erkennen Sie die Nutzungsbedingungen an.

\section{Terms of use}

This document is published under following Creative Commons-License: http://creativecommons.org/licenses/by-sa/4.0/deed.en - You may copy, distribute and transmit, adapt or exhibit the work or its contents in public and alter, transform, or change this work as long as you attribute the work in the manner specified by the author or licensor. New resulting works or contents must be distributed pursuant to this license or an identical or comparable license.

By using this particular document, you accept the above-stated conditions of use.

\section{Kontakt / Contact:}

\section{peDOcs}

DIPF | Leibniz-Institut für Bildungsforschung und Bildungsinformation Informationszentrum (IZ) Bildung

E-Mail: pedocs@dipf.de

Internet: www.pedocs.de 


\section{von}

Lehrpersonen

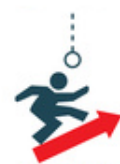

i 


\section{Impressum}

\section{Haushalt in Bildung \& Forschung (HiBiFo)}

Herausgeber:

HaBiFo Haushalt in Bildung und Forschung e.V.

Prof. ${ }^{\text {in }}$ Dr. ${ }^{\text {in }}$ Kirsten Schlegel-Matthies, Universität Paderborn

\section{Redaktion:}

Ass.-Prof. ${ }^{\text {in }}$ Dr. ${ }^{\text {in }}$ Claudia Maria Angele, Universität Wien

Werner Brandl M.A., München

Prof. ${ }^{\text {in }}$ Dr. ${ }^{\text {in }}$ Angela Häußler, PH Heidelberg

Prof. ${ }^{\text {in }}$ Dr. ${ }^{\text {in Julia Kastrup, FH Münster }}$

Prof. ${ }^{\text {in }}$ Dr. ${ }^{\text {in }}$ Kirsten Schlegel-Matthies, Universität Paderborn

Claudia Wespi lic. phil., PH Luzern

redaktion@hibifo.de•www.hibifo.de

\section{Erscheinen und Bezugsbedingungen:}

Die Zeitschrift erscheint 4 x jährlich mit einem Jahresumfang von rd. 400 Seiten (Print und Online). Abonnements verlängern sich automatisch um ein Jahr. Abonnement-Kündigungen bitte schriftlich an den Verlag. Kündigungsfrist bis drei Monate zum Jahresende.

Das digitale Angebot, alle Informationen zum Abonnement sowie zu Einzelausgaben finden Sie auf https://hibifo.budrich-journals.de.

Bestellungen bitte an den Buchhandel oder an:

Verlag Barbara Budrich GmbH, Stauffenbergstr. 7, D-51379 Leverkusen-Opladen

Tel.: +49 (0)sssss171.344.594, Fax:+49 (0)2171.344.693, info@budrich.de

www.budrich-journals.de $\bullet$ www.budrich.de

Aktuelle Mediadaten/Anzeigenpreisliste: HiBiFo 818 vom 01.02.2018

Fachliche Betreuung des Heftes: Claudia Wespi

Titelbild: Werner Brandl; Bildnachweis $(C)$ trueffelpix/fotolia

Heft 4, Jg. 8, 2019

\section{(C) 2019 Verlag Barbara Budrich GmbH Opladen • Berlin • Toronto}

Die Zeitschrift sowie alle in ihr enthaltenden Beiträge sind urheberrechtlich geschützt. Jede Verwertung, die nicht ausdrücklich vom Urheberrechtsgesetz zugelassen ist, bedarf der vorherigen Zustimmung des Verlags. Dies gilt insbesondere für Vervielfältigungen, Übersetzungen, Mikroverfilmungen und die Einspeicherung und Verarbeitung in elektronischen Systemen.

Namentlich gekennzeichnete Beiträge geben nicht in jedem Fall die Meinung der Redaktion wieder.

Druck: paper \& tinta, Warschau

Printed in Europe

ISSN 2193-8806

Online-ISSN 2196-1662 
Claudia Wespi

Editorial.....

Joanna Hellweg

Das Kohärenzgefühl als Einflussfaktor bei der Förderung

professioneller Kompetenz von Lehrpersonen im Studium 3

Werner Brandl

Professionalisierung und Professionalität - Anmerkungen

zur Bedeutung und Entwicklung von Lehrkompetenz 18

Elisabeth Eichelberger \& Susanna Holliger

SchülerInnenfirma Repair Café - Kompetenzen

entwickeln und anwenden.

Ute Bender

Videobasierte Fallanalysen als Beitrag zur Professionalisierung

von angehenden Lehrpersonen in der Ernährungs- und Verbraucherbildung -

am Beispiel der Führung von Unterrichtsgesprächen

Claudia Wespi \& Markus Steiner

„Wirtschaft entdecken“ - eine computergestützte Lernumgebung

mit Lernpotenzial für Lernende und Lehrpersonen

Silke Bartsch, Katharina Henke, Heike Müller \& Isabelle Penning

Verbraucherkompetenzen für morgen durch Lehrkräftebildung heute:

Professionalisierung von Lehrpersonen in der Verbraucherbildung 90

Sinah Gerdes \& Corinne Senn

Mit Classcraft motivierend

Wirtschaft-Arbeit - Haushalt (WAH) unterrichten 104

Jana Markert

Biografische Selbstreflexion in der Lehramtsausbildung: Erarbeitung der eigenen Bildungsbiografie mittels der systemischen Methode des Lebensfluss-Modells

Mareike Bröcheler

Rezension: Reformprojekt Care Work - Professionalisierung

der beruflichen und akademischen Ausbildung 
Silke Bartsch, Katharina Henke, Heike Müller \& Isabelle Penning

\section{Verbraucherkompetenzen für morgen durch Lehrkräftebildung heute: Professionalisierung von Lehrpersonen in der Verbraucherbildung}

Verbraucherbildung ist bundesweit vielfältig in curricularen Vorgaben verankert. Fachfremde Lehrpersonen benötigen Professionalisierungsangebote, um die Verbraucherbildung als Querschnittsaufgabe in ihren Fächern implementieren zu können. Erkenntnisse aus der Professionalisierungsforschung werden daher im Beitrag in Bezug auf das cLEVER-Projekt zusammengetragen, um sie für Fortbildungsangebote nutzbar zu machen.

Schlüsselwörter: Verbraucherbildung, Consumer Literacy, Professionalisierung, Lehrerbildung, Fortbildung

\section{Consumer competences for tomorrow through teacher education today: Professionalizing consumer education teachers}

Consumer education is anchored in a variety of curricular guidelines throughout Germany. Teachers from outside the subject area need professionalization offers in order to be able to implement consumer education as a cross-sectional task in their subjects. Findings from professionalization research will, therefore, be compiled in the article relating to the cLEV ER-project to make them usable for further training courses.

Keywords: consumer education, consumer literacy, professionalization, teacher training, continuing education

\section{Verbraucherbildung und Professionalisierung}

Durch die Digitalisierung steigt die Komplexität der globalisierten Waren- und Dienstleistungswelt aus rechtlicher und wirtschaftlicher Sicht. Personalisierte, dynamische Preisgestaltung bei Internetangeboten, Influencer Marketing, eingeschränkte Unabhängigkeit von kommerziellen Bewertungsportalen, Erkennen von Fake Shops oder Datenschutzverletzungen sind nur Beispiele, die die alltäglichen Herausforderungen für Verbraucherinnen und Verbraucher in jedem Alter zeigen. Eine weitere Facette ist die Bildung für nachhaltige Entwicklung. So hebt das nationale Programm für nachhaltigen Konsum, das 2016 beschlossen wurde, die Rolle der Verbraucherbildung für nachhaltige Entwicklung hervor (BMU, 2019, S. 26). Folgt man dem idealisierten Verbraucherleitbild, dann sollen sich gebildete Menschen mündig und eigenverantwortlich gegenüber der Weltgesellschaft, ihrer Um- 


\section{Verbraucherbildung und Lehrkräfteprofessionalisierung}

und Mitwelt und für sich selbst verhalten. Trotz der notwendigen Differenzierung des fiktiven Leitbildes (vgl. Kenning \& Wobker, 2013), orientiert sich Bildung insbesondere am Ziel der Mündigkeit (z.B. KMK, 2013, 2015). In diesem Beitrag wird daher der Begriff der Mündigkeit und (Eigen-)Verantwortlichkeit genutzt, wohlwissend um dessen Limitierungen.

Wenngleich auch die verschiedenen Grenzen der Verbraucherbildung als Teil des Verbraucherschutzes (vgl. Heidbrink \& Schmidt, 2011; Grunwald, 2012) hier nicht diskutiert werden können, ist offensichtlich, dass die dafür notwendige Consumer Literacy eine Grundbildung erforderlich macht, die nicht allein einem lebensweltorientierten Ankerfach aufgebürdet werden kann, sondern bestenfalls auch eine schulische Querschnittsaufgabe ist (vgl. VK BW, 2015; Bartsch \& Häußler, 2018). Dem entspricht der Beschluss der Kultusministerkonferenz zur Verbraucherbildung (2013):

Die Verbraucherbildung hat die Entwicklung eines verantwortungsbewussten Verhaltens als Verbraucherinnen und Verbraucher zum Ziel, indem über konsumbezogene Inhalte informiert wird und Kompetenzen im Sinne eines reflektierten und selbstbestimmten Konsumverhaltens erworben werden. Dabei geht es vor allem um den Aufbau einer Haltung, die erworbenen Kompetenzen im Zusammenhang mit Konsumentscheidungen als mündige Verbraucherinnen und Verbraucher heranzuziehen und zu nutzen. (KMK, 2013, S. 1)

Die KMK sieht Fortbildungen von Lehrpersonen in den Themenfeldern der Verbraucherbildung als eine langfristige Maßnahme zur Stärkung der Verbraucherbildung an Schulen (KMK, 2013, S. 4). Dabei ist für die unterrichtliche Umsetzung der Verbraucherbildung als Querschnittsthema ein Fortbildungsbedarf auf verschiedene Zielebenen feststellbar: 1. Verbraucherbildung als Teil der Allgemeinbildung anerkennen (vgl. auch Bartsch, Bauer \& Müller, 2018), 2. Bildungsauftrag für die eigenen Unterrichtsfächer erkennen und 3. Unterstützung bei der fachlichen und fachdidaktischen Umsetzung (ebd.). Darüber hinaus weist die KMK auf die Möglichkeit der Verhältnisorientierung, z.B. als Teil der Schulentwicklung hin (KMK, 2013, S. 3f.).

\section{Schulische Verbraucherbildung in Deutschland}

Mit dem Beschluss der KMK (2013) zur Verbraucherbildung ist eine bundesweite schulische Verankerung angestoßen worden. Das ist ein Novum in der rund 60jährigen Geschichte der Verbraucherbildung. Die Bundesländer - gemäß der föderalen Struktur ist die Ausgestaltung Ländersache (Art. 30 GG) - setzen den Beschluss auf vielfältige Weise in sehr unterschiedlichem Tempo um (KMK, 2015). Es lassen sich grundsätzliche Ansätze identifizieren: Verbraucherbildung als Schulfach oder Verbraucherbildung als Querschnittsaufgabe aller Fächer mit oder ohne Ankerfach. Während der Beschluss der KMK vorsieht, Verbraucherbildung 


\section{Verbraucherbildung und Lehrkräfteprofessionalisierung}

,in den Unterricht eines oder mehrerer Unterrichtsfächer“ zu integrieren (KMK, 2013, S. 4), präferieren die Vereinten Nationen eindeutig den Querschnittsansatz: "Consumer education should, where appropriate, become an integral part of the basic curriculum of the educational system, preferably as a component of existing subjects" (A/RES/70/186;43). Das schließt ein Leit- oder Ankerfach jedoch nicht aus und stellt deren Notwendigkeit auch nicht in Frage (vgl. VK BW, 2015).

Zum Beispiel wurde in Schleswig-Holstein bereits 2009/2010 die Fachlösung umgesetzt und das Fach Haushaltslehre in Verbraucherbildung (VBB) umbenannt (MBWK, o. A.). VBB ist ein eigenständiges Unterrichtsfach in der Sekundarstufe I und an Förderzentren ab Klassenstufe 5 oder 7. Aktuell werden die Fachanforderungen überarbeitet, begleitet von Forderungen der Verbraucherzentrale Schleswig-Holstein und der Verbraucherzentrale Bundesverband e.V. u. a zur umfassenden Qualifizierung in allen Phasen der Lehrerbildung (VZ SH \& VZBV, 2019).

Beispiele für den Querschnittansatz finden sich in Baden-Württemberg, Berlin und Brandenburg. In Berlin und Brandenburg ist Verbraucherbildung eine von 13 Querschnittsaufgaben (sog. fächerübergreifende Themen). Der gemeinsame Rahmenlehrplan für die Klassen 1 bis 10 wird seit 2017 in Berlin, nicht in Brandenburg, um den Orientierungs- und Handlungsrahmen Verbraucherbildung ergänzt (vgl. SenBFJ, 2016). Für Brandenburg wird 2019 vom Arbeitskreis schulische Verbraucherbildung der „Maßnahmenkatalog zur Unterstützung der Schulen bei der Umsetzung der curricularen Anforderungen von Verbraucherbildung" erarbeitet. Außerdem werden Fortbildungen für Lehrpersonen im Projekt EVeLaB (https://evelabildung.-wordpress.com) im Rahmen der Qualitätsoffensive Schulverpflegung angeboten.

In Baden-Württemberg wurde mit dem Bildungsplan 2016 Verbraucherbildung als eine von sechs fächerübergreifenden Leitperspektiven als Bildungsauftrag für allgemeinbildende Schulen eingeführt (KM, 2016). Um Lehrpersonen zu unterstützen, fördert das Ministerium für Ländlichen Raum und Verbraucherschutz BadenWürttemberg (MLR) seit 2016 das cLEVER-Projekt (www.leitperspektiveverbraucherbildung.de). Die Ergebnisse der Begleitforschung verdeutlichen, dass der Bildungsauftrag Verbraucherbildung noch nicht von allen Lehrpersonen wahrgenommen wird. So gibt es u. a Lehrpersonen, denen noch zu wenig bewusst ist, dass sie Verbraucherbildung im Fachunterricht umsetzen müssen und auch solche, die kaum Vorstellungen dazu haben, wie die Umsetzung im Fachunterricht gelingen kann (Bartsch et al., 2018; Müller, 2018). Erschwerend kommt hinzu, dass die Leitperspektiven in den vorhandenen Schulbüchern nicht systematisch berücksichtigt werden (Bartsch et al., 2018). Daher werden im Projekt cLEVER u. a Fortbildungen für Multiplikatorinnen und Multiplikatoren sowie Pädagogische Tage an Schulen durchgeführt, bei denen gemeinsam mit den Lehrpersonen Ideen für die Umsetzung im Fachunterricht entwickelt werden. Weitere Unterstützungsangebote für Lehrpersonen in Baden-Württemberg sind der Verbraucherschutzpreis für 


\section{Verbraucherbildung und Lehrkräfteprofessionalisierung |}

Schulen, der 2019 bereits zum sechsten Mal gemeinsam vom MLR zusammen mit dem Kultusministerium und der Verbraucherkommission Baden-Württemberg (VK BW) ausgeschrieben wurde, sowie ein ebenfalls vom MLR gefördertes Bildungsprojekt der Verbraucherzentrale Baden-Württemberg e.V. mit dem Schwerpunkt Unterrichtsmaterialien. Mit dem sog. Qualitätskonzept erneuert das Kultusministerium aktuell das Fortbildungssystem in Baden-Württemberg. Offen ist, ob Verbraucherbildung darin systematisch verankert wird, um die Umsetzung des Bildungsauftrags zu sichern.

Zusammenfassend zeigt sich, dass die curriculare Verankerung nach wie vor lückenhaft ist - auch in Bundesländern wie Schleswig-Holstein, weil beispielsweise VBB nicht an Grundschulen, Gymnasien und beruflichen Schulen etabliert ist. Bei der Querschnittsaufgabe Verbraucherbildung droht diese im schulischen Alltag schnell „unter den Tisch zu fallen“, zudem Verbraucherbildung selten prüfungsrelevant ist. Neben der curricularen Verankerung stellt sich die Frage der unterrichtlichen Umsetzung. Die Implementierung der Verbraucherbildung ist also nach wie vor nicht abgeschlossen.

Bei der Frage nach der Qualität in Schul- und Unterrichtspraxis lassen sich zwei Probleme feststellen. Zum einen existieren keine strukturell verankerten Qualitätssicherungsmaßnahmen zur Umsetzung curricularer Vorgaben. Zum anderen stellt sich die Frage, inwieweit Lehrpersonen auf die Aufgabe vorbereitet werden, Verbraucherbildung im Unterricht aber auch im Setting Schule (Bartsch \& Häußler, 2018) umzusetzen. Letzteres ist kein neues Problem: Lehrpersonen in der Verbraucherbildung waren schon immer eine äußerst heterogene Gruppe.

A large number of unidentifiable 'consumer educators' teach elements of Consumer Education without initial training and in-service training, maybe without knowing that what they are teaching is Consumer Education. Another group of teachers, e. g. teachers of Home Economics, Work Studies ('Arbeitslehre') and sometimes Social Studies, have had initial training at universities or teacher training colleges and invest effort and time in retraining and material development. (Steffens, 1992, S. 17)

Die Gruppe derer, die sich schon in der ersten Phase der Lehrerbildung mit Verbraucherbildung auseinandergesetzt hat, ist klein, was sich einerseits an vergleichsweise wenigen Fachlehrpersonen in der haushaltsbezogenen Bildung zeigt (vgl. Heseker \& Beer, 2004) und andererseits darauf zurückzuführen ist, dass sich Verbraucherbildung nur vereinzelt in den Studien- und Prüfungsordnungen des Lehramts im Allgemeinen wiederfindet (vgl. Schlegel-Matthies, 2016, S. 102). „Verbraucherbildung kann jedoch auf angemessenem Niveau nur von gut ausgebildeten professionellen Kräften unterrichtet werden, die fachlich und methodisch mit der Domäne vertraut sind" (ebd.). Auch die UN fordert von Mitgliedsstaaten daher die Organisation oder Förderung von Fortbildungsprogrammen u. a für Lehrpersonen, damit diese in die Lage versetzt werden, sich an der Durchführung von Verbraucherinformations- und Bildungsprogrammen beteiligen zu können 
Verbraucherbildung und Lehrkräfteprofessionalisierung

(A/RES/70/186;48). Für die schul- und unterrichtspraktische Implementierung von Verbraucherbildung besteht ein Bedarf, Lehrpersonen in allen Phasen der Lehrerbildung für Verbraucherbildung zu qualifizieren.

\section{Verbraucherkompetenzen fördern durch Fortbildung von Lehrpersonen}

\subsection{Lehrkräfteprofessionalisierung im Kontext der Verbraucherbildung}

Lehrpersonenfortbildungen in Themenfeldern der Verbraucherbildung können dazu beitragen, diese an Schulen zu stärken (KMK, 2013, S. 4). Für die Teilnahme an Fortbildungen gibt es keine bundesweit einheitliche Regelung. In BadenWürttemberg, wo das hier vorgestellte cLEVER-Projekt umgesetzt wird, sind Lehrpersonen gemäß der Landeslaufbahnverordnung zur Teilnahme an dienstlichen Fortbildungen sowie zur individuellen Fortbildung verpflichtet. Gleichzeitig gibt es keine genauen Vorgaben zum Umfang der Teilnahme (vgl. Johannmeyer, Drahmann \& Cramer, 2019). Damit obliegt die Entscheidung ob, wie oft und wie intensiv Fortbildungsangebote wahrgenommen werden den Lehrpersonen selbst (vgl. Riedinger, 2010, S. 9f.; vgl. Johannmeyer et al., 2019, S. 24). Sowohl diese Freiheit als auch andere Rahmenbedingungen, wie beispielweise die Komplexität der Fortbildungsstruktur, können die Professionalisierung in dieser dritten Phase der Lehrerbildung erschweren (vgl. Cramer, Johannmeyer \& Drahmann 2019). Die oftmals ausbleibende Verzahnung der Fortbildung mit den anderen Phasen der Lehrerbildung, fehlende finanzielle Mittel, mangelnde Bedarfsorientierung sowie unzureichende einheitliche und verbindliche Qualitätsstandards sind weitere Herausforderungen der Lehrerfortbildung in Deutschland (vgl. Johannmeyer et al., 2019; Koch, 2016; vgl. auch Göb, 2017).

Im Rahmen des cLEVER-Projekts konnte ein Fortbildungsbedarf zur Umsetzung der Leitperspektive Verbraucherbildung in den Unterricht aller Fächer festgestellt werden (vgl. Bartsch et al., 2018). Der Bildungsauftrag der Leitperspektive Verbraucherbildung (KM, 2016) wird von den Lehrpersonen teilweise gar nicht als solcher wahrgenommen, als Überforderung bewertet oder nicht vollständig erfüllt (ebd.). Verbraucherbildung steht hier beispielhaft für die Herausforderungen bei der Implementierung von Querschnittsthemen.

\subsection{Lehrpersonenfortbildungen und ihre Wirkungsebenen}

Während im Bereich der Wirksamkeit von Lehrpersonenfortbildungen zahlreiche Studien vorliegen, gibt es zu Gründen und Motiven der Lehrpersonen für eine Fortbildungsteilnahme noch wenige Ergebnisse (vgl. Koch, 2016, S. 12). Zwar 


\section{Verbraucherbildung und Lehrkräfteprofessionalisierung}

liegen aus den Bundesländern Ansätze zur Berichterstattung zu Fortbildungen vor (DVLfB, 2018, S. 125). Jedoch sind mit diesen Daten keine Rückschlüsse auf das individuelle Fortbildungsverhalten von Lehrpersonen möglich und auch eine systematische Auswertung der Daten zur Prüfung und Sicherung der Fortbildungsqualität ist u. a aufgrund der Heterogenität der Daten ebenfalls nicht durchführbar (ebd.).

Die Frage, ob das Fortbildungssystem den Kompetenzerwerb von Lehrpersonen angemessen unterstützt, sodass diese den heutigen und zukünftigen Herausforderungen in ihrer Profession konstruktiv begegnen können, ist Gegenstand verschiedener Studien (vgl. Hanisch, 2017, S. 468). Die Beantwortung dieser und damit zusammenhängenden Forschungsfragen ist besonders aufgrund der Komplexität und Fülle der Einflussvariablen und der damit einhergehenden methodischen Herausforderung in der Forschung schwierig (vgl. Lipowsky \& Rzejak, 2015, S. 11). Dennoch wurden in der Wirksamkeitsforschung verschiedene Wirkungsebenen definiert (vgl. Lipowsky, 2010; Lipowsky \& Rzejak, 2012; Huber \& Radisch, 2010). Sie stimmen darin überein, dass die Teilnahme von Lehrpersonen an Fortbildungsmaßnahmen weitreichende Auswirkungen haben kann und verorten einen „Fortbildungserfolg“" auf vier Ebenen (Abbildung 1).

Ebene 1

Akzeptanz und Zufriedenheit der teilnehmenden Lehrpersonen

Ebene 2

Erweiterung des Lehrerwissens, Weiterentwicklung der Überzeugungen und Orientierungen u.a.

\section{Ebene 3}

Weiterentwicklung der Unterrichtsqualität

\section{Ebene 4}

Förderung des Lernens der Schülerinnen und Schüler

Abb. 1: Wirkungsebenen des Angebots-Nutzungsmodell zur Erklärung des Lernens von Lehrpersonen im Rahmen von Fortbildungen (nach Liposwky, 2019, S. 145; siehe auch Lipowsky \& Rzejak, 2017; Lipowsky, 2014)

Auf einer ersten Wirkungsebene kann sich der Fortbildungserfolg durch die Reaktionen der teilnehmenden Lehrpersonen zeigen. Positive Rückmeldungen von Lehrpersonen nach der Fortbildungsteilnahme lassen aber keine sicheren Schlüsse auf Veränderungen im unterrichtlichen Handeln zu oder gar auf positive Veränderungen des Lernerfolgs der Schülerinnen und Schüler. Diese auch als „Evalua- 
Verbraucherbildung und Lehrkräfteprofessionalisierung

tionsebene" bezeichnete Ebene kann somit von den folgenden Ertragsebenen isoliert betrachtet werden. Auf der zweiten Ebene kann der Fortbildungserfolg anhand des Lernzuwachses der Lehrpersonen bemessen werden. Dieser Lernzuwachs umfasst sowohl kognitive als auch affektiv-motivationale Dimensionen. Auf der dritten Ebene kann Fortbildungserfolg an Veränderungen im unterrichtlichen Handeln der teilnehmenden Lehrpersonen festgestellt werden. Die Teilnahme an Lehrerfortbildungen kann sich bis auf die vierte Ebene, die Ebene der Schülerinnen und Schüler, auswirken und deren Lernleistungen positiv beeinflussen. Huber und Radisch (2010) erwähnen außerdem mögliche Auswirkungen auf das Kollegium und auf die Schulebene. Diese zusätzliche Ebene ist für die Verbraucherbildung lohnenswert, weil sie das Setting Schule als Erfahrungsraum einbezieht und Verbraucherkompetenzen aller Akteure in Schulen fördern kann (Bartsch \& Häußler, 2018). Forschungen zu Angeboten der Lehrerfortbildung gibt es für die Verbraucherbildung kaum, insbesondere nicht auf den Wirkungsebenen zwei bis vier nach dem Angebots-Nutzungs-Modell (Liposwky, 2019, S. 145; siehe auch Lipowsky \& Rzejak, 2017; Lipowsky, 2014).

\section{4 cLEVER: Implikationen für Fortbildungen zur Verbraucherbildung}

Ziel des cLEVER-Projekts ist, Lehrpersonen zu unterstützen, die Leitperspektive Verbraucherbildung in allen Schularten, Fächern und Klassenstufen umzusetzen, u. a durch Fortbildungen. Im Folgenden werden Erkenntnisse der Professionalisierungsforschung zusammengetragen und in Bezug auf das cLEVER-Projekt konkretisiert.

\subsection{Gemeinsam Schule und Unterricht entwickeln}

Die Zusammenarbeit von Lehrpersonen wird in vielen Fortbildungsmaßnahmen angestrebt, da sich hier positive Effekte im Hinblick auf den Erwerb handlungsnaher Kompetenzen und auf die Teilnahmemotivation gezeigt haben (vgl. Lipowsky \& Rzejak, 2017, S. 383). Bezüglich Schulentwicklungsprozesse wird konstatiert, dass Steuergruppen stark an der Entwicklung des Unterrichts mitwirken, wobei jedoch die Einflüsse der Steuergruppen und deren Effektivität bislang wenig erforscht wurden (vgl. Bonsen \& Berkemeyer, 2014). In Bezug auf die langfristige Implementierung von Verbraucherbildung an Schulen scheint es sinnvoll, wenn Schulleitungen die Lehrpersonenqualifizierung mithilfe eines gesamtschulischen Fortbildungsplans systematisch unterstützen. Das cLEVER-Projekt kann über gezielte Formate, wie z. B Pädagogische Tage, die Gründung von Entwicklungsteams innerhalb von Schulen fördern, an dem beispielsweise Lehrpersonen unterschiedlicher Fächer teilhaben und ein schulinternes, fächerverbindendes Curriculum ge- 


\section{Verbraucherbildung und Lehrkräfteprofessionalisierung}

meinsam entwickeln. Solchen Angeboten, ,an denen größere Teile eines Kollegiums teilnehmen können", wird Bedeutung für die Nachhaltigkeit der Fortbildung beigemessen (Cramer et al., 2019, S. 11; vgl. auch Johannmeyer et al., 2019). Die an den Fortbildungen teilnehmenden Lehrpersonen können als Multiplikatorinnen und Multiplikatoren innerhalb ihrer professionellen Kontexte wirken. Um eine systematisch verankerte Verbraucherbildung an Schulen und deren Entwicklung zu fördern, sind auch Möglichkeiten der schulischen Profilbildung zu eruieren. Schulen haben die Möglichkeit spezifische Fachprofile zu entwickeln, was in verschiedenen Bereichen gängig ist. In ähnlicher Weise könnte ein Profil „Verbraucherbildung" für Schülerinnen und Schüler attraktiv sein und insgesamt Ressourcen für eine fächerverbindende, systematische und dauerhafte Implementierung freisetzen.

\subsection{Forschung und Lehre verbinden}

In der ersten Phase der Lehrerbildung ist die Verknüpfung von Forschung und Lehre ein zentrales Kennzeichen. Auch in der dritten Phase ist das Einbringen von Forschungsergebnissen aus der Lehr- und Lern- und Unterrichtsforschung in Fortbildungen gewinnbringend (Lipowsky \& Rzejak, 2017, S. 385). Durch die universitäre Verankerung des cLEVER-Projekts und die im Projekt geleistete Begleitforschung ist die Implementierung von Forschungsergebnissen in universitären und externen Qualifizierungsmaßnahmen leicht möglich.

\subsection{Verschränkung von Input, Erprobungs- und Reflexionsphase}

Erfolgreiche Fortbildungen sind anwendungsbezogen und keine „Drive-byWorkshops“ (Bonsen \& Berkemeyer, 2014, S. 923). Sie sind nah am Berufsalltag ausgerichtet und eine Verschränkung von Input, Erprobung und Reflexion ist systematisch verankert (vgl. Lipowsky \& Rzejak, 2017, S. 390f.). In den Fortbildungen des cLEVER-Projekts können die Input-Phasen an Fallbeispielen aus dem Unterrichtsalltag ausgerichtet werden, da sich das situierte Lernen ebenfalls als wirksam erwiesen hat (ebd.). Nach einer Sensibilisierung für das Thema Verbraucherbildung und der Erarbeitung sowohl von fachwissenschaftlichen Grundlagen und fachdidaktischen Zugängen werden die Lehrpersonen gebeten, in Teams fachspezifische Unterrichtsideen zu entwickeln. Aufgrund der aktuellen Projektstruktur werden einmalig stattfindende Veranstaltungen als Impuls gesetzt mit der Möglichkeit, vertiefende Workshops anzufragen. Perspektivisch können eine Erprobungs- und Auswertungsphase implementiert werden, z.B. durch eine Dokumentation der Erprobungsphasen mithilfe von Videomitschnitten oder Protokollen, die in anschließenden Reflexionsphasen systematisch ausgewertet werden können. Dabei kann ein Wechsel zwischen direkten Kolleginnen und Kollegen aus der gleichen Schule und schulübergreifenden Fachkolleginnen und -kollegen variierende Per- 
Verbraucherbildung und Lehrkräfteprofessionalisierung

spektiven ermöglichen und zur konstruktiven Weiterentwicklung der Unterrichtskonzepte beitragen.

\subsection{Dauer der Fortbildung}

Obwohl Liposwky und Rzejak (2017) davon ausgehen, dass die Konzeption von Fortbildungen wesentlich bedeutsamer ist als der Fortbildungsumfang, wird die Wirksamkeit von einmalig stattfindenden Fortbildungen (sog. „One-ShotFortbildungen“) angezweifelt (S. 391f.). Ein linear positiver Zusammenhang lässt sich jedoch nicht nachweisen (Timperley, Wilson, Barrar \& Fung, 2007, S. 75). Der Versuch, die zuvor genannten wirksamen Merkmale bei der Konzeption einfließen zu lassen, führt fast automatisch zu Fortbildungsformaten, die über EinTagesangebote hinausgehen. Insbesondere bei der Verbraucherbildung, die als Leitperspektive alle Lehrpersonen betrifft, ist eine umfangreiche Qualifizierung notwendig. Mit Blick auf den bundesweiten Fortbildungsbedarf könnten Anschlussprojekte auch die Umsetzung von Blended-Learning-Formaten prüfen. Die systematische Verschränkung zwischen Präsenzterminen und E-Learning-Phasen könnten den Transfer der bislang in Baden-Württemberg durchgeführten Fortbildungen in andere Bundesländer erleichtern sowie der Vereinbarkeit mit den weiteren Dienstaufgaben der Lehrpersonen durch eine zeitliche Flexibilisierung zuträglich sein.

\subsection{Unterstützungssysteme}

Koch (2016) fordert vor dem Hintergrund der hohen Anzahl fachfremd unterrichtender Lehrkräfte im Bereich der ökonomischen Bildung, „, dass aus den Qualifizierungsmaßnahmen heraus bzw. in direkter Verknüpfung mit ihnen weitergehende Unterstützungssysteme etabliert werden, auf die dauerhaft zugegriffen werden kann“ (S. 19). Aufgrund der bislang geringen Implementierung von Verbraucherbildung in allen Fächern in den ersten beiden Phasen der Lehrpersonenbildung, ist seine Forderung hier begründet übertragbar und mit Nachdruck zu unterstützen. Im cLEVER-Projekt wird die Qualifizierung von Lehrpersonen über Fortbildungen für Multiplikatorinnen und Multiplikatoren sowie Pädagogische Tage für Schulen angestrebt. Zusätzlich werden Handreichungen zur „Verbraucherbildung im Fachunterricht" mit Hintergründen zur Verbraucherbildung, exemplarischen Unterrichtsbausteinen und Verweisen auf Partnerschaften in der Verbraucherbildung entwickelt und, neben weiteren für die Zielgruppe relevanten Informationen, die über die Projektwebseite zur Verfügung gestellt werden.

Im weiteren Projektverlauf könnte geprüft werden, inwiefern die Bereitstellung der exemplarischen Unterrichtsbausteine als Open Educational Resources mit offener Lizenz zielführend ist. Unter einer solchen Lizenz wäre die freie Nutzung, Weiterentwicklung und -verbreitung durch Lehrpersonen und pädagogisch Interes- 


\section{Verbraucherbildung und Lehrkräfteprofessionalisierung}

sierte möglich. Sprachliche Übersetzungen der Unterrichtsbausteine könnten beispielsweise die weltweite Verbreitung der Materialien begünstigen und damit der global hohen Relevanz des Themas Verbraucherbildung entgegenkommen.

Mit der Bereitstellung von Unterrichtsbausteinen wurde auch den Erwartungen der Lehrpersonen entsprochen, die im cLEVER-Projekt erhoben wurden und sich mit vergleichbaren Studien decken, in denen sich die Lehrpersonen schnell umsetzbare Impulse und Unterrichtsmaterialien wünschen (vgl. Wolf, Göbel-Lehnert \& Chroust, 1999; Jäger \& Bodensohn, 2007, zitiert nach Lipowsky, 2014, S. 530f.). Lipowsky zeigt jedoch eine Diskrepanz zwischen den Erwartungen von Lehrpersonen an Fortbildungen und den Erkenntnissen zur Wirksamkeit auf: ,[...] die von Lehrern immer wieder formulierten Erwartungen an Fortbildungen [...] rücken vor allem die schnelle und kurzfristige Verwertbarkeit in den Vordergrund, während aus Sicht der Forschung fruchtbare Lernaktivitäten und nachhaltige Lernprozesse von Lehrpersonen eher langfristiger Natur sind und durchaus harte und anstrengende Arbeit darstellen" (Lipowsky, 2014, S. 530f.; vgl. auch Göb, 2017). Dementsprechend wird im cLEVER-Projekt die Bereitstellung von exemplarischen Unterrichtsmaterialien vor dem Hintergrund des hohen Qualifizierungsbedarfes zwar als notwendig erachtet, jedoch lediglich als eine Maßnahme betrachtet, welche die umfassende Lehrpersonenqualifizierung in Form von Fortbildungen flankiert. Entsprechend der Transferforschung wird mit Hilfe von Konzepten und Materialpaketen lediglich das explizite Wissen transferiert (vgl. Höfer \& Rolff, 2015, S. 612). Insbesondere im Hinblick auf die Verbraucherbildung ist ebenfalls zu untersuchen, wie implizite Wissensbestände transferiert werden können.

\section{Fazit und Ausblick}

Verbraucherbildung ist eines von mehreren Querschnittsthemen mit hoher Relevanz, für das ein hoher Qualifizierungsbedarf bei den Lehrpersonen besteht. Um Verbraucherbildung dauerhaft und auf qualitativ hohem Niveau zu stärken, müssen Ressourcen in ausreichendem Umfang langfristig bereitgestellt werden. Die vorhandenen Ressourcen sollten den teilnehmenden Lehrpersonen und engagierten Schulen zugutekommen, aber auch genutzt werden, um Unterstützungsstrukturen weiter auszubauen und zu verstetigen.

Darüber hinaus sind die Forschungsdesiderate im Bereich der Verbraucherbildung und der Professionalisierung zu bearbeiten. Um die als wirksam erachtete fachliche Tiefe und die Fokussierung auf die Lernenden (Lipowsky \& Rzejak, 2017, S. 388f.) zu ermöglichen, sind weitere Studien erforderlich, die beispielsweise Schülervorstellungen zu Verbraucherthemen erheben und Aspekte des conceptual change berücksichtigen. Zusätzlich sind für ein qualitätsgesichertes Fortbildungskonzept eine kontinuierliche Evaluation und eine Weiterentwicklung der $\mathrm{Maßnahmen} \mathrm{notwendig.} \mathrm{Ob} \mathrm{eine} \mathrm{bundesweite} \mathrm{Adressierung} \mathrm{von} \mathrm{Lehrpersonen} \mathrm{vor}$ 


\section{Verbraucherbildung und Lehrkräfteprofessionalisierung}

dem Hintergrund des Föderalismus im Kontext der bestehenden differierenden Fortbildungsorte und -anbietern möglich ist und wie diese gelingen könnte, ist zu eruieren. $\mathrm{Zu}$ bedenken ist, dass die curriculare Verankerung der Verbraucherbildung in den Bundesländern unterschiedlich verläuft.

\section{Literatur}

Bartsch, S. \& Häußler, A. (2018). Verbraucherbildung ist mehr als Unterricht. In SchVw Baden-Württemberg, 3/2018, 82-85. (Nachdruck: SchVW Spezial 18(3), 103-106).

Bartsch, S., Bauer, A.-K. \& Müller, H. (2018). Transfer der Leitperspektive Verbraucherbildung in den Unterricht. Schlussbericht. Karlsruhe.

https://www.leitperspektive-verbraucherbildung.de/2018/02/01/erfolgreicherabschluss-von-clever-1-start-von-folgeprojekt-clever-2/

Bonsen, M. \& Berkemeyer, N. (2014). Lehrerinnen und Lehrer in Schulentwicklungsprozessen. In E. Terhart, H. Bennewitz \& M. Rothland (Hrsg.), Handbuch der Forschung zum Lehrerberuf (2. Aufl.) (S. 920-936). Münster, New York: Waxmann.

BMU - Bundesministerium für Umwelt, Naturschutz, Bau und Reaktorsicherheit, BMJV \& BMEL (Hrsg.) (2019, 3. akt. Aufl.). Nationales Programm für nachhaltigen Konsum Gesellschafticher Wandel durch einen nachhaltigen Lebensstil (3. Aufl.). Berlin.

https://www.bmu.de/fileadmin/Daten_BMU/Pools/Broschueren/

nach_haltiger_konsum_broschuere_bf.pdf

Cramer, C., Johannmeyer, K. \& Drahmann, M. (Hrsg.). (2019). Fortbildungen von Lehrerinnen und Lehrern in Baden-Württemberg. Stuttgart: GEW.

DVLfB - Deutscher Verein zur Förderung der Lehrerinnen- und Lehrerfortbildung e.V. (2018). Recherchen für eine Bestandsaufnahme der Lehrkräftefortbildung in Deutschland. Ergebnisse des Projektes Qualitätsentwicklung in der Lehrkräftefortbildung. Teil 1. forum Lehrerfortbildung, 47.

https://www.fachportal-paedagogik.de/literatur/vollanzeige.html?FId=1155672

Göb, N. (2017). Professionalisierung durch Lehrerfortbildung: Wie wird der Lernprozess der Teilnehmenden unterstützt? Die Deutsche Schule, 109(1), 9-27.

Grunwald, A. (2012). Ende einer Illusion. Warum ökologisch korrekter Konsum uns nicht retten wird. München: oekom.

Hanisch, R. (2017). Die Fortbildung von Lehrkräften vor aktuellen gesellschaftlichen und bildungspolitischen Herausforderungen. In H.-G. Kotthoff \& T. Leuders (Hrsg.), Lehrkräftefortbildung: Herausforderungen, Umsetzungen, Wirkungen (S. 467-480). Wien: Böhlau.

Heidbrink, L. \& Schmidt, I. (2011). Das Prinzip der Konsumentenverantwortung Grundlagen, Bedingungen und Umsetzungen verantwortlichen Konsums. In L. 


\section{Verbraucherbildung und Lehrkräfteprofessionalisierung |}

Heidbrink, I. Schmidt \& B. Ahaus (Hrsg.), Die Verantwortung des Konsumenten. Über das Verhältnis von Markt, Moral und Konsum. Frankfurt a. M.: Campus.

Heseker, H. \& Beer, S. (2004). Ernährung und ernährungsbezogener Unterricht in der Schule. Bundesgesundheitsblatt, Gesundheitsforschung, Gesundheitsschutz, 47, 240-245.

Höfer, C. \& Rolff, H.-G. (2015). Transfer von Unterrichtsentwicklung. In H.-G. Rolff (Hrsg.), Handbuch Unterrichtsentwicklung (S. 609-635). Weinheim, Basel: Beltz.

Huber, S. \& Radisch, F. (2010). Lehrerfort- und -weiterbildung. Ein Rahmenmodell für Forschung und Evaluation. In B. Schwarz, P. Nenniger \& R. S. Jäger (Hrsg.), Erziehungswissenschaftliche Forschung - nachhaltige Bildung. Beiträge zur 5. DGfE-Sektionstagung ,Empirische Bildungsforschung“/AEPFKBBB im Frühjahr 2009 (S. 204-222). Landau: Empirische Pädagogik e.V.

Jäger, R. S. \& Bodensohn, R. (2007). Die Situation der Lehrerfortbildung im Fach Mathematik aus Sicht der Lehrkräfte. Ergebnisse einer Befragung von Mathematiklehrern.

https://dzlm.de/files/uploads/17_01_07_mathematiklehrerbefragung.pdf

Johannmeyer, K., Drahmann, M. \& Cramer, C. (2019). Stand der Forschung zu Fortbildungen von Lehrerinnen und Lehrern sowie deren Rahmenbedingungen. In C. Cramer, K. Johannmeyer \& M. Drahmann (Hrsg.), Fortbildungen von Lehrerinnen und Lehrern in Baden-Württemberg. Stuttgart: GEW.

Kenning, P. \& Wobker, I. (2013). Ist der „mündige Verbraucher“ eine Fiktion? Ein kritischer Beitrag zum aktuellen Stand der Diskussion um das Verbraucherleitbild in den Wirtschaftswissenschaften und der Wirtschaftspolitik. Zeitschrift für Wirtschafts- und Unternehmensethik, 14(2), 282-300.

KM - Ministerium für Kultus, Jugend und Sport Baden-Württemberg. (2016). Bildungsplan 2016. http://www.bildungsplaene-bw.de/,Lde/Startseite

KMK (2013). Verbraucherbildung an Schulen. Beschluss der Kultusministerkonferenz vom 12.09.2013

https://www.kmk.org/fileadmin/veroeffentlichungen_beschluesse/2013/2013_0 9_12-Verbraucherbildung.pdf

KMK (2015). Bericht zur Verbraucherkompetenz von Schülerinnen und Schülern. https://www.kmk.org/fileadmin/Dateien/veroeffentlichungen_beschluesse/2015 /2015_12_03-Bericht-Verbraucherbildung-VSMK-KMK.pdf

Koch, M. (2016). Die dritte Phase der Lehrerqualifizierung. Entwicklungsaufgabe im deutschen Bildungssystem und in der ökonomischen Bildung. Zeitschrift für ökonomische Bildung, (4), 1-35. http://www.zfoeb.de/2016_4/2016_4_koch_— dritte phase_der_lehrerqualifizierung.pdf

MBWK - Ministerium für Bildung, Wissenschaft und Kultur des Landes Schleswig-Holstein (MBWK). (o. A.). Verbraucherbildung. 


\section{Verbraucherbildung und Lehrkräfteprofessionalisierung}

https://lehrplan.lernnetz.de/index.php?wahl=149

Müller, H. (2018). Themen sind nah am (Konsum-) Alltag der Schüler/innen. bildung \& wissenschaft, 07-08, 24-26. https://d.pr/JezKOn+

Lipowsky, F. (2010). Lernen im Beruf. Empirische Befunde zur Wirksamkeit von Lehrerfortbildung. In F. Müller, A. Eichenberger, M. Lüders \& J. Mayr (Hrsg.), Lehrerinnen und Lehrer lernen. Konzepte und Befunde zur Lehrerfortbildung (S. 51-70). Münster: Waxmann.

Lipowsky, F. (2014). Theoretische Perspektiven und empirische Befunde zur Wirksamkeit von Lehrerfort- und -Weiterbildung. In E. Terhart, H. Bennewitz \& M. Rothland (Hrsg.), Handbuch der Forschung zum Lehrerberuf (S. 511541) (2. Aufl.). Münster u. a: Waxmann.

Lipowsky, F. (2019). Wie kommen Befunde der Wissenschaft in die Klassenzimmer? - Impulse der Fortbildungsforschung. In C. Donie, F. Foerster, M. Obermayr, A. Deckwerth, G. Kammermeyer, G. Lenske, M. Leuchter \& A. Wildemann (Hrsg.), Grundschulpädagogik zwischen Wissenschaft und Transfer (S. 144-161). (Jahrbuch Grundschulforschung, Bd. 23). Wiesbaden: VS.

Lipowsky, F. \& Rzejak, D. (2012). Lehrerinnen und Lehrer als Lerner - Wann gelingt der Rollentausch? Merkmale und Wirkungen effektiver Lehrerfortbildungen. In D. Bosse, L. Criblez \& T. Hascher (Hrsg.), Reform der Lehrerbildung in Deutschland, Österreich und der Schweiz. Teil 1: Analysen, Perspektiven und Forschung (S. 235-253). Immenhausen: Prolog.

Lipowsky, F. \& Rzejak, D. (2015). Das Lernen von Lehrpersonen und Schülern/innen im Fokus. Was zeichnet wirksame Lehrerfortbildung aus? In A. Grimm (Hrsg.), Was Wirklich Wirkt!? Effektive Lernprozesse und Strukturen in Lehrerfortbildung und Schulentwicklung (S. 11-49). Rehburg-Loccum, [Hildesheim]: Evangelische Akademie Loccum.

Lipowsky, F. \& Rzejak, D. (2017). Fortbildungen für Lehrkräfte wirksam gestalten. Erfolgsversprechende Wege und Konzepte aus Sicht der empirischen Bildungsforschung. Bildung und Erziehung, 70(4), 379-399.

Riedinger, A. (2010). Lehrerfortbildung in Baden-Württemberg. (Schriftenreihe Studien zur Berufspädagogik, 36). Hamburg: Kovač.

Schlegel-Matthies, K. (2016). Konsum, Ernährung, Gesundheit - Neues Schulfach, Querschnittsaufgabe oder Leitlinie? SchVW Spezial, 18(3), 100-102.

SenBFJ - Senatsverwaltung für Bildung, Jugend und Wissenschaft (2016). Orientierungs- und Handlungsrahmen für das übergreifende Thema Verbraucherbildung gemäß dem im November 2015 in Kraft gesetzten Rahmenlehrplan für die Jahrgangsstuften 5-10. Berlin: Senatsverwaltung.

Steffens, H. (1992). Consumer Education in Germany. In E. Rientjes, G. Hellman, \& H. M. Wachters-Patmore (Hrsg.), Consumer Education - Theory, Practice and Developments. Seminar Proceedings (S. 17-19). The Hague: IOCU Regional Office for Europe and North America. 


\section{Verbraucherbildung und Lehrkräfteprofessionalisierung}

Timperley, H., Wilson, A., Barrar, H. \& Fung, I. (2007). Teacher Professional

Learning and Development. Best Evidence Synthesis Iteration [BES]. Wellington, New Zealand: Ministry of Education. http://www.oecd.org/education/school/48727127.pdf.

VK BW - Verbraucherkommission Baden-Württemberg (2015). Stellungnahme zur Bildungsplanreform 2016. Stellungnahme, 39/2015. https://t1p.de/1 riv

VZ SH \& VZBV - Verbraucherzentrale Schleswig-Holstein e.V. \& Verbraucherzentrale Bundesverband e.V. (2019). Fachanforderungen Verbraucherbildung. Gemeinsame Stellungnahme. Anlass: Anhörung zum Entwurf der Fachanforderungen (Stand 25.01.2019) durch das Ministerium für Schule und Berufsbildung des Landes Schleswig-Holstein, geplante Einführung: Schuljahr 2019/2020. Kiel/ Berlin VZ SH/VZBV. https://www.vzbv.de/sites/default/files/downloads/2019/04/12/ stellungnahme_vbzv_vzsh_fachanforderungen_verbraucherbildung_2019.pdf

Wolf, W., Göbel-Lehnert, U. \& Chroust, P. (1999). Fortbildung der Lehrinnen und Lehrer. Eine Bilanz ihrer Formen und Wirkungen anhand empirischer Untersuchungen. Die Deutsche Schule, 91, 451-467.

\section{Verfasserinnen}

Prof. ${ }^{\text {in }}$ Dr. ${ }^{\text {in }}$ Silke Bartsch

Katharina Henke

Heike Müller

Dr. ${ }^{\text {in }}$ Isabelle Penning

Technische Universität Berlin

Institut für Berufliche Bildung und Arbeitslehre

Fachdidaktik Arbeitslehre

Marchstr. 23

D-10587 Berlin

E-Mail: silke.bartsch@tu-berlin.de

Internet: www.alfa.tu-berlin.de

cLEVER-Projekt: www.leitperspektive-verbraucherbildung.de 